\title{
PREVALENCIA DE PANCREATITIS AGUDA EN EL EMBARAZO*
}

\author{
Drs. Daniel Ríos-Cruz ${ }^{1}$, Joaquín Valerio-Ureña ${ }^{1}$, Fátima Santiago-Pérez ${ }^{1}$ \\ ${ }^{1}$ Departamento de Cirugía General. Hospital de Alta Especialidad de Veracruz. \\ México.
}

\begin{abstract}
\section{Prevalence of acute pancreatitis in pregnancy}

Objetive: The aim of this study was to determine the prevalence and characteristics of acute pancreatitis during pregnancy in the Hospital de Alta Especialidad de Veracruz in Mexico. Methods: A retrospective, descriptive study of all pregnant patients diagnosed with acute pancreatitis from January to December 2013 treated in our hospital was conducted. Results: 4.478 records of pregnant women were reviewed, 6 had acute pancreatitis during pregnancy, it corresponds to 1 in 746.33 pregnant patients. The age of our patients was $28 \pm 6.30$ years (range 15-32 years). The weeks of gestation had a mean of $16.3 \pm 8.7$ weeks (range 10-30 weeks). In all cases was mild pancreatitis secondary to gallstones disease. The time of hospital stay was $8.79 \pm 4.26$ days (range 7-18 days). Five patients underwent laparoscopic cholecystectomy; a patient did not accept surgery. One fetal death was presented. Conclusion: Acute pancreatitis during pregnancy is a rare complication. The ideal time to perform a cholecystectomy is the second trimester. The prognosis is generally good for both mother and the product.
\end{abstract}

Key words: Acute pancreatitis, pregnancy.

\section{Resumen}

Objetivo: Determinar la prevalencia y características de la pancreatitis aguda durante el embarazo en el Hospital de Alta Especialidad de Veracruz, México. Material y Métodos: Se realizó un estudio retrospectivo, descriptivo de todas las pacientes embarazadas diagnosticadas con pancreatitis agudas de enero tratadas en este Hospital. Resultados: Se revisaron 4.478 expedientes de mujeres gestantes, de las cuales 6 presentaron pancreatitis aguda durante su embarazo, esto corresponde a una de cada 746,33 pacientes embarazadas. La edad de nuestros pacientes fue de $28 \pm 6,30$ años (rango 15-32 años). Las semanas de gestación tuvieron una media de 16,3 \pm 8,7 semanas (rango 10-30 semanas). En todos los casos la pancreatitis fue leve y de origen biliar. El tiempo de estancia intrahospitalaria fue de 8,79 4,26 días (rango 7-18 días). Cinco pacientes fueron sometidas a colecistectomía laparoscópica y una no aceptó procedimiento quirúrgico. Se presentó un óbito. Conclusión: La pancreatitis aguda durante el embarazo es una complicación poco frecuente. El tiempo ideal para realizar una colecistectomía es el segundo trimestre. El pronóstico es, en general, bueno tanto para la madre como para el producto.

Palabras clave: Pancreatitis aguda, embarazo.

\footnotetext{
*Recibido el 7 de abril de 2014 y aceptado para publicación el 28 de mayo de 2014.

Los autores no refieren conflictos de interés.

Correspondencia: Dr. Daniel Ríos-Cruz jobzon@hotmail.com
} 


\section{Introducción}

El abdomen agudo continúa siendo un reto para todo médico que toma parte en el cuidado de una mujer embarazada. Se estima que la incidencia del abdomen agudo durante el embarazo es de 1 en 500635 embarazos $^{1}$ siendo la apendicitis la causa más común, que corresponde al $25 \%$ de las indicaciones quirúrgicas no obstétricas durante la gestación. La incidencia de la pancreatitis varía aproximadamente de 1 en 1.000 a 1 en 10.000 nacimientos $^{3}$. Los cambios fisiológicos asociados al embarazo incrementan el riesgo de presentar pancreatitis. Si bien la incidencia parece ir en aumento, los casos de pancreatitis aguda continúan siendo poco frecuentes. En México pocos datos reportados sobre este tema. El objetivo de este estudio fue determinar la prevalencia y características de la pancreatitis aguda durante el embarazo en el Hospital de Alta Especialidad de Veracruz, México.

\section{Material y Método}

Se trata de un estudio retrospectivo y observacional. Se revisaron los expedientes clínicos de todos los pacientes egresados de los servicios de Cirugía General y Gineco-obstetricia del Hospital de Alta Especialidad de Veracruz en México durante el período de enero a diciembre de 2013. Se seleccionaron los expedientes clínicos de todas las mujeres embarazadas diagnosticadas con pancreatitis de cualquier etiología y gravedad. Para establecer el diagnóstico de pancreatitis se utilizaron los criterios del American College of Gastroenterology que establece que el diagnóstico de pancreatitis se obtiene por dos de los siguientes parámetros: clínicos (dolor abdominal en epigastrio o hipocondrio derecho, transfixiante, irradiado en hemicinturón y constante), bioquímicos (elevación mayor a tres veces del límite superior normal de los niveles de amilasa y lipasa séricas) y de imagen (hallazgos compatibles con pancreatitis en tomografía abdominal computada y/o resonancia magnética) ${ }^{4}$. Se tomaron en cuenta todos los eventos de pancreatitis aguda que se presentaron (no solamente el número de pacientes) con el fin de que las recidivas se incluyeran en la prevalencia de la enfermedad. Se excluyeron a pacientes cuyos expedientes clínicos estuvieran incompletos. De los expedientes se obtuvo la edad de los pacientes al momento de la pancreatitis aguda, las semanas de gestación, la etiología de la pancreatitis, así como su gravedad utilizando los criterios de Atlanta y el score de Marshall modificado, así como puntuación de APACHE $\mathrm{II}^{5}$, los días de estancia intrahospitalaria, la evolución del embarazo, las recidivas, el tratamiento empleado así como la morbilidad y mortalidad tanto de la madre como del producto.

Se utilizó estadística descriptiva (media, porcentaje, tasa) con el programa SPSS 20.

\section{Resultados}

Durante el período de estudio de 12 meses (enero-diciembre de 2013) se atendieron en el hospital a 4.478 mujeres embarazadas.

Se revisaron los expedientes de estas mujeres gestantes, de las cuales 6 presentaron pancreatitis aguda durante su embarazo, una de ellas presentó dos cuadros de pancreatitis por lo que en total se tuvieron 7 eventos de pancreatitis.

Se utilizó ultrasonido de hígado y vía biliar como estudio de imagen para ayudar a establecer el origen de la pancreatitis; por este método de imagen, todas las pacientes presentaron evidencia de litos de diversas dimensiones únicamente en la vesícula biliar y no así en el conducto colédoco. Ninguna de las pacientes presentó indicación para que fuese sometida a tomografía computada.

Se obtuvo la tasa de pancreatitis aguda durante el embarazo al relacionar el número de pacientes con pancreatitis durante la gestación con el número total de pacientes embarazadas atendidas en el hospital. Esto corresponde al $0,133 \%$, es decir, una de cada 746,33 pacientes embarazadas que se atendieron en el hospital tuvo pancreatitis. Tomando en cuenta el total de eventos de pancreatitis en nuestra población (7 eventos) la prevalencia fue de 0,0015 .

La edad de nuestros pacientes fue de $28 \pm 6,30$ años (rango 15-32 años), únicamente tuvimos una paciente adolescente embarazada con pancreatitis. Las semanas de gestación tuvieron una media de $16,3 \pm 8,7$ semanas (rango 10-30 semanas), una paciente en el primer trimestre, tres en el segundo trimestre y dos en el tercer trimestre. En todos los casos la pancreatitis fue leve y de origen biliar con una puntuación de APACHE II de $4 \pm 1,3$ (rango 3-6 puntos). El tiempo de estancia intrahospitalaria fue de 8,79 \pm 4,26 días (rango 7-18 días) (Tabla 1).

Las determinaciones bioquímicas de amilasa y lipasa, en todos los casos, cumplieron con el criterio para diagnóstico de pancreatitis. Los niveles de glucosa, creatinina y leucocitos se mantuvieron dentro de parámetros normales. En cuanto al perfil hepático hubo un incremento generalizado de la bilirrubina total con un patrón colestásico (incremento a expensas de la bilirrubina directa) sin ir más allá de dos veces su valor normal, las demás enzimas se mantuvieron en parámetros normales. La presión arterial sistólica se mantuvo por arriba de los $90 \mathrm{mmHg}$ en todos los casos y el índice de oxigenación fue mayor a 400 en todas las pacientes (Tabla 2). 
Tabla 1. Características generales de los pacientes estudiados. Los datos generales son expresados en medias, rangos y porcentajes

\begin{tabular}{|ll|}
\hline Datos generales & Valores \\
\hline Pacientes & 6 \\
Edad & $28 \pm 6,30$ años (rango 15-32) \\
Semanas de gestación & $16,3 \pm 8,7$ semanas (rango 10-30) \\
Etiología biliar & $6(100 \%)$ \\
\hline APACHE II & $4 \pm 1,3$ puntos (rango 3-6) \\
Gravedad leve & $6(100 \%)$ \\
\hline Estancia hospitalaria & $8,79 \pm 4,26$ días (rango 7-18) \\
Recidiva & $1(16,6 \%)$ \\
\hline Tratamiento quirúrgico & $5(83.33 \%)$ \\
Morbilidad materna & 0 \\
\hline Mortalidad materna & 0 \\
\hline Morbilidad fetal & 0 \\
Mortalidad fetal & $1(16,6 \%)$ \\
\hline
\end{tabular}

Cinco pacientes fueron sometidas a evento quirúrgico. Tres de las pacientes fueron llevadas a colecistectomía laparoscópica sin complicaciones una vez remitido el cuadro de pancreatitis, esto fue durante el segundo trimestre. Una paciente requirió conversión del procedimiento laparoscópico a procedimiento abierto. Una paciente que presentó pancreatitis en el tercer trimestre fue llevada a cole- cistectomía laparoscópica posterior a la resolución del embarazo. Una paciente no aceptó procedimiento quirúrgico.

Como se mencionó, una paciente tuvo recurrencia de la pancreatitis. No se presentó morbilidad ni mortalidad materna durante el período de estudio. Una paciente de 32 años que presentó pancreatitis durante el primer trimestre, con cuadro remitido y resolución quirúrgica durante el segundo trimestre acudió a su control prenatal a las 37 semanas de gestación refiriendo ausencia de movimientos fetales y el embarazo terminó en un óbito.

\section{Discusión}

La incidencia anual de la pancreatitis aguda es de 5 a 80 casos por cada $100.000^{3}$. En mujeres embarazadas se reporta desde 1 en 1.000 a 3 en 10.000 embarazos $^{3,6,7}$.

En nuestro país existen pocos trabajos que traten la temática. Un estudio realizado por el Dr. DíazPizarro $^{8}$ hace 10 años, nos muestra el panorama que existía en un hospital de concentración, reportando que el $0,0445 \%$ de las pacientes embarazadas atendidas en ese centro hospitalario presentaron pancreatitis aguda, esto es, una paciente de cada 2.249 embarazadas.

Recientemente González-González 9 y cols., reportan 10 casos de pancreatitis en mujeres embarazadas en un período de 6 años en un hospital de la ciudad de Monterrey.

Nuestros datos revelan que en nuestro medio

Tabla 2. Datos bioquímicos y fisiológicos de los pacientes estudiados. Los datos son expresados en medias y rangos

\begin{tabular}{|lll|}
\hline Sustancia & Valor & \\
Amilasa & $1.061,5 \pm 473,3 \mathrm{U} / \mathrm{L}$ & (rango 778-2.081) \\
Lipasa & $1.315,4 \pm 463 \mathrm{U} / \mathrm{L}$ & (rango 974-2.076) \\
Leucocitos & $10,3 \pm 110^{3} / \mathrm{mm}^{3}$ & (rango 8,9-11,5) \\
Bilirrubina total & $1,4 \pm 0.3 \mathrm{mg} / \mathrm{dL}$ & (rango 0,9-1,8) \\
$\quad$ Bilirubina directa & $0,8 \pm 0,27 \mathrm{mg} / \mathrm{dL}$ & (rango 0,5-1,3) \\
Bilirrubina indirecta & $0,47 \pm 0,09 \mathrm{mg} / \mathrm{dL}$ & (rango 0,4-0,6) \\
Transaminasa glutámico oxalacética & $40,02 \pm 7,6 \mathrm{U} / \mathrm{L}$ & (rango 32-50) \\
Transaminasa glutámico pirúvica & $37,3 \pm 3,9 \mathrm{U} / \mathrm{L}$ & (rango 33-45) \\
Fosfatasa alcalina & $129 \pm 31 \mathrm{U} / \mathrm{L}$ & (rango 89-170) \\
Gammaglutamil transpeptidasa & $44,5 \pm 9,62 \mathrm{U} / \mathrm{L}$ & (rango 33-60) \\
Creatinina & $0,7 \pm 0,14 \mathrm{mg} / \mathrm{dL}$ & (rango 0,54-0,98) \\
Glucosa & $106 \pm 15,1 \mathrm{mg} / \mathrm{dL}$ & (rango 87-127) \\
\hline Presión arterial sistólica & $104 \pm 11,9 \mathrm{mmHg}$ & (rango 90-120) \\
Índice de oxigenación & $442 \pm 11,9$ & (rango 429-461) \\
\hline
\end{tabular}


una de cada 746,33 mujeres embarazadas presenta pancreatitis aguda. Se puede argumentar esta situación debido a los hábitos alimentarios y al estilo de vida que se tienen actualmente. Estas cifras son similares a las de un estudio realizado en Shanghai, China en un período de 5 años donde se reporta que una de cada 708 mujeres embarazadas cursaron con pancreatitis ${ }^{10}$.

Se ha postulado que mientras más avanzado se encuentra el embarazo, es más probable que la paciente presente pancreatitis ${ }^{11}$. Sun y cols. ${ }^{6}$, analizaron a pacientes embarazadas con pancreatitis durante un período de 11 años y reportan que el $50,7 \%$ de los casos de pancreatitis se presentaron en el tercer trimestre y únicamente el 7,3\% en el primer trimestre. Similar a lo reportado por Zhang y cols. ${ }^{12}$, quien muestra que el $79 \%$ de las pacientes, estudiadas durante 6 años, correspondían a embarazos del tercer trimestre. Sin embargo, en nuestra casuística es más frecuente durante el segundo trimestre.

Todos nuestros casos correspondieron a pancreatitis leve y esto se puede relacionar a la semana de gestación en la que se presentó. En la literatura internacional se reporta la relación que existe entre la gravedad de la pancreatitis y las semanas de gestación. Se ha observado que la gran mayoría de los casos de pancreatitis aguda grave en el embarazo se presentan en el tercer trimestre ${ }^{6,13,14}$. La pancreatitis durante el primer trimestre se asocia a sufrimiento fetal y durante el tercer trimestre se asocia a trabajo de parto prematuro, preeclampsia, síndrome de HELLP e inclusive muerte materna y/o muerte fetal $^{15,16}$.

La etiología de la pancreatitis asociada al embarazo es similar a la que se presenta en mujeres no embarazadas. En todos los casos, el origen de la pancreatitis fue biliar lo cual se presenta acorde a la literatura internacional cuando se refiere que la causa más común de pancreatitis aguda en la población en general es debido a problemas de litiasis vesicular y más aún en embarazadas en quienes, debido a los cambios fisiológicos propios del embarazo, la secreción hepática de colesterol se encuentra incrementada en comparación a las sales biliares y fosfolípidos llevando a una bilis super-saturada; adicionalmente el volumen de la bilis contenida en la vesícula biliar se encuentra incrementada tanto en el ayuno como en el postprandio ${ }^{3,17}$.

La formación de lodo biliar y litos se encuentra fuertemente asociado al número de embarazos ${ }^{18}$. Únicamente una paciente era primigesta. Dos pacientes, cuartigestas y tres pacientes, tercigestas.

Las complicaciones que se pueden presentar pueden ser divididas en morbimortalidad materna y fetal. Se ha descrito la mortalidad materna en $<1 \%$; el parto pretérmino en $15-32 \%$ de los casos; la mor- talidad perinatal en $10-20 \%{ }^{19}$. Las complicaciones como pseudoquistes, hemorragias, necrosis, peritonitis generalizada, síndrome de distress respiratorio del adulto, coagulación intravascular diseminada y muerte son menos frecuentes que en mujeres no embarazadas $^{20}$. El único caso lamentable en nuestra serie fue la pérdida del producto en una paciente. Sin embargo, esto sucedió en el tercer trimestre y el cuadro de pancreatitis se presentó en el primero. No existen estudios disponibles que asocien estas complicaciones con la presentación temprana del cuadro de pancreatitis. En apariencia, el embarazo posterior a la resolución de la pancreatitis y la cirugía, transcurrió de manera normal y el expediente no revela alguna anormalidad durante ese período.

Aunque es conocido que parte del tratamiento para la pancreatitis de origen biliar es la colecistectomía y, si existe coledocolitiasis, la colangiopancreatografía retrógrada endoscópica (CPRE), es necesario conocer el tiempo oportuno para poder realizarla sobre todo en pacientes embarazadas.

En los inicios de la era laparoscópica, el embarazo fue considerado una contraindicación relativa para realizar una colecistectomía, principalmente por la falta de conocimientos sobre el efecto del $\mathrm{CO}_{2}$ sobre el feto. El temor de realizarla se basaba en riesgo potencial de aborto y malformaciones si ésta se practicaba en el primer trimestre o un parto pretérmino si se realizaba durante el tercer trimestre $^{21}$. Está bien establecido que el segundo trimestre del embarazo es el tiempo ideal para llevar a cabo un procedimiento laparoscópico ya que el período de organogénesis ha concluido y los riesgos, tanto para el feto como para la madre, son mínimos, aunado a que el tamaño del útero no interfiere con la visualización del campo quirúrgico y la frecuencia de abortos espontáneos es menor que el primer trimestre $^{22-24}$. Cuatro de nuestras pacientes fueron sometidas a colecistectomía laparoscópica en el segundo trimestre y una de ellas tuvo que ser convertida a cirugía abierta debido al gran proceso inflamatorio y adherencial de las estructuras vecinas hacia vesícula y vía biliar. Una paciente esperó a la resolución de su embarazo para ser operada y una más no aceptó tratamiento quirúrgico.

Tocante a la CPRE, existen estudios ${ }^{25,26}$, que muestran la seguridad y eficacia de este procedimiento durante el embarazo bajo el argumento de que la radiación a la que el feto se expone durante el estudio es muy por debajo del límite máximo permitido $(0,005 \mathrm{~Gy})$ para evitar complicaciones al producto. En nuestras pacientes no fue necesario llevar a cabo algún procedimiento dirigido a la vía biliar, ya que previo y durante la cirugía ninguna de ellas evidenció coledocolitiasis.

La pancreatitis aguda durante el embarazo es una 
complicación poco frecuente. El conocimiento de los cambios fisiológicos propios del embarazo es útil para entender porqué una mujer gestante tiene más posibilidades de presentarla. El soporte médico es parte fundamental del tratamiento que se complementa con la cirugía cuando su origen es biliar. El tiempo ideal para realizar una colecistectomía es el segundo trimestre y el pronóstico es, en general, bueno tanto para la madre como para el producto.

\section{Referencias}

1. Unal A, Sayharman SE, Ozel L, Unal E, Aka N, Titiz $\mathrm{I}$, et al. Acute abdomen in pregnancy requiring surgical management: a 20-case series. European Journal 88 of Obstetrics \& Gynecology and Reproductive Biology 2011;159:87-90.

2. Long SS, Long C, Lai H, Macura KJ. Imaging strategies for right lower quadrant pain in pregnancy. Am J Roentgenol. 2011;196:4-12.

3. Pitchumoni C, Yegneswaran B. Acute Pancreatitis in Pregnancy. World J Gastroenterol. 2009;15:5641-6.

4. Tenner S, Baillie J, DeWitt J, Vege SS. American College of Gastroenterology Guideline: Management of Acute Pancreatitis. Am J Gastroenterol. 2013;108:140015.

5. Banks PA, Bollen TL, Dervenis C, Gooszen HG, Johnson CD, Sarr MG, et al. Classification of acute pancreatitis-2012: revision of the Atlanta classification and definitions by international consensus. Gut 2013;62:102-11

6. Sun L, Li W, Geng Y, Shen B, Li J. Acute Pancreatitis in Pregnancy. Acta Obstet Gynecol Scand. 2011;90:671-6.

7. Qihui C, Xiping Z, Xianfeng D. Gastroenterol Res Pract. 2012;27:19-25.

8. Díaz-Pizarro JI, Mijares-García JM, LE CárdenasLailson. Prevalencia de la pancreatitis aguda durante el embarazo y puerperio. Cir Gen. 2003;25:152-7.

9. González-González JA, Castañeda-Sepúlveda R, Martínez-Vázquez MA, García-Compean D, Flores-Rendón AR, Maldonado-Garza HJ, et al. Rev Gastroenterol Mex. 2012;77:167-73.

10. Hua-ping LI, Ya-juan H, Xuan C. Acute pancreatitis in pregnancy: a 6-year single center clinical experience. Chinese Medical Journal 2011;124:2771-5.

11. Ramin KD, Ramin SM, Richey SD, Cunningham FG. Acute pancreatitis in pregnancy. Am J Obstet Gynecol. 1995; 173:187-91.

12. Zhang D, Huang Y, Yan L, Phu A, Ran X, Li S. Thirty- eight cases of Acute Pancreatitis in Pregnancy: A 6-year Single Center Retrospective Analysis. J Huazhong Univ Sci Technol. [Med Sci]. 2013;33:361-7.

13. Ducarme G, Chatel P, Alves A, Hammel P, Luton D. Management of necrotizing pancreatitis in the third trimester of pregnancy. Arch Gynecol Obstet. 2009;279:561-3.

14. Tang SJ, Rodríguez-Frías E, Singh S, Mayo MJ, Jazrawi SF, Sreenarasimhaiah J, et al. Acute pancreatitis during pregnancy. Clin Gastroenterol Hepatol. 2010;8:85-90.

15. Malangoni MA. Gastrointestinal surgery and pregnancy. Gastroenterol Clin North Am. 2003;32:181-200.

16. Sun Y, Fan C, Wang S. Clinical analysis of 16 patients with acute pancreatitis in the third trimester of pregnancy. Int J Clin Exp Pathol. 2013;6:1696-701.

17. Maringhini A, Ciambra M, Baccelliere P, Raimondo M, Orlando A, Tine F, et al. Biliary sludge and gallstones in pregnancy: incidence, risk factors, and natural history. Ann Intern Med. 1993;119:116-20.

18. Pandey M, Shukla VK. Lifestyle, parity, menstrual and reproductive factors and risk of gallbladder cancer. Eur J Cancer Prev. 2003;12:269-72.

19. Eddy JJ, Gideonsen MD, Song JY, Grobman WA, O'Halloran P. Pancreatitis in Pregnancy: a 10 year retrospective of 15 Midwest Hospitals. Obstet Gynecol. 2008;112:1075-81.

20. Gilbert A, Patenaude V, Abenhaim HA. Acute pancreatitis in pregnancy: a comparison of associated contidions, treatments and complications. J Perinat Med. 2014 (en prensa).

21. Chiappetta Porras LT, Nápoli ED, Canullán CM, Quesada BM, Roff HE, Alvarez Rodríguez J. Minimally InvasiveManagement of Acute Biliary Tract Disease during Pregnancy. HPB Surgery 2009, Article ID 829020, 3 pages doi:10.1155/2009/829020.

22. Date RS, Kaushal M, Ramesh A. A review of the management of gallstone disease and its complications in pregnancy. Am J Surg. 2008;196:599-608.

23. McKellar DP, Anderson CT, Boynton CJ, Peoples JB. Cholecystectomy during pregnancy without fetal loss. Surg Gynecol Obstet. 1992;174:465-8.

24. Biscette S, Yoost J, Hertweck P, Reinstine J. Laparoscopy in Pregnancy and the Pediatric Patient. Obstet Gynecol Clin N Am. 2011;38:757-76.

25. Smith I, Gaidhane M, Goode A, Kahaleh M. Safety of endoscopic retrograde cholangiopancreatography in pregnance: Fluoroscopy time and fetal exposure, does it matter?. World J Gastrointest Endosc. 2013; 5:148-53.

26. Yarmuch J. Cirugía y embarazo. Rev Chil Cir. 2010;62:520-4. 